\title{
Unintended consequences of coverage laws targeting cancer drugs
}

\author{
Maximilian Salcher-Konrad and Huseyin Naci \\ Department of Health Policy, London School of Economics and Political Science, London, United \\ Kingdom.
}

"Why do cancer drugs get such an easy ride?" (Light and Lexchin, 2015). This question was originally asked about regulatory standards for approval, but it may be just as pertinent to cancer drugs' coverage in insurance plans. In a case study in Massachusetts, Leopold and colleagues identified several state laws targeting the coverage of cancer drugs in health insurance programs. Such "onco-exceptionalism" in coverage policy poses challenges for payers confronted with high-cost drugs with limited evidence on clinical benefits (Leopold et al., 2020). Leopold et al.'s nuanced analysis reveals how payers navigate the complex terrain between regulatory and payment policy.

Do cancer drugs warrant special treatment? The majority of new cancer drugs are indicated for the treatment of patients with reduced life expectancy and significant unmet therapeutic needs. Both the public and patients favor special funding for new therapies targeting these populations if new treatment options offer important benefits (MacLeod, Harris and Mahal, 2016). However, the therapeutic benefit of cancer drugs is often uncertain due to limitations in the evidence base supporting their market entry. Over the past two decades, most cancer drugs have benefitted from FDA's expedited development and review programs. Drugs approved under these programs enter the market faster, but at the cost of less complete data. Only about half of clinical trials supporting FDA's cancer drug approvals between 20152017 were randomized, $5 \%$ had active comparator arms, and $80 \%$ had surrogate measures as their primary endpoints (Zhang et al., 2020).

The quantity and quality of data available on new drugs are shaped primarily by FDA's evidentiary standards. However, pharmaceutical manufacturers also react to signals given through insurance legislation. For example, research and development activity on cancer drugs has increased following the implementation of the Federal Medicare Part D program in 2006, which mandated inclusion of all FDAapproved cancer drugs in private plan formularies offering Part D benefits (Blume-Kohut and Sood, 2012). Cancer drugs now account for the single largest category of new drug approvals, and there are more products than ever in the development pipeline (Aitken et al., 2019).

This upward trend in research and development activity has not translated into better evidence. An important unintended consequence of coverage laws targeting cancer drugs has been the further erosion of incentives for manufacturers to generate higher-quality evidence.

Despite considerable uncertainties in their effectiveness, most cancer drugs now cost more than \$100,ooo per patient per year. Health insurance coverage is therefore essential for patient access to these therapies. However, payers' negotiating powers are limited when coverage of cancer drugs is mandatory. In the current environment, payers are not able to negotiate meaningful discounts for cancer drugs (Hernandez et al., 2020). High drug prices affect patient access: despite expanded Medicare Part D coverage, increases in drug prices have led to higher out-of-pocket expenses for cancer patients (Dusetzina, Huskamp and Keating, 2019).

What are potential solutions? Payers interviewed by Leopold and colleagues recommended adopting closed formularies. Indeed, plans can manage uncertainty by selectively covering drugs that provide meaningful benefits to patients. Interestingly, Massachusetts had requested in 2018 the authority to establish a closed formulary for its Medicaid program, which was later rejected by the Centers for Medicare and Medicaid Services (Sommers and Kesselheim, 2018). 
Determining drug coverage in closed formularies should be based on comparative clinical effectiveness or cost-effectiveness considerations, and ideally, both. Such evidence-based approaches can eliminate (or at least minimize) the arbitrary variation observed in coverage decisions among commercial insurers in the US (Chambers et al., 2018), and ensure patients have access to drugs with proven therapeutic value.

Payers in the US can learn from other high-income countries that have decades of experience with using these approaches. For example, in Germany, new drugs can enter the market after regulatory approval, but coverage through statutory health insurance is only determined after an assessment of the drugs' added therapeutic benefit compared to standard of care. Drugs that do not provide any added therapeutic benefit according to comparative clinical effectiveness assessments may still be available to patients if manufacturers agree to set their prices at comparable levels to other drugs that offer similar benefits. In a recent analysis, the German Institute for Quality and Efficiency in Healthcare (IQWiG) determined that fewer than half of new cancer drugs provided major or considerable benefits over available alternatives (Wieseler, McGauran and Kaiser, 2019). Only a small proportion of drugs can therefore secure higher prices than existing options.

The German experience also demonstrates how coverage decisions can be directly linked to the quality of available evidence. IQWiG's comparative clinical assessments explicitly penalize drugs with an uncertain evidence base. For example, drugs with studies that measure surrogate endpoints, lack randomization, or use inadequate control groups are not eligible to demonstrate added benefit over existing treatments. This approach to creating a closed formulary has been effective. Over the past decade, Germany's national formulary has granted coverage to all drugs with added benefit while market withdrawals have been limited to drugs with no proven benefit (Stern et al., 2019).

Other countries also consider cost-effectiveness as an explicit benchmark for drug coverage. Costeffectiveness analyses evaluate whether a new drug's incremental benefits are worth their extra cost. For over two decades, the National Institute for Health and Care Excellence (NICE) in England has relied on cost-effectiveness analyses to determine which drugs to recommend for routine use in the English National Health Service. NICE generally recommends drugs for coverage if they cost less than between $£ 39,000(\$ 59,611)$ and $£ 44$, ooo $(\$ 67,254)$ per quality-adjusted life-year (QALY) gained (Dakin et al., 2015). In the US, the role of the independent Institute for Clinical and Economic Review (ICER) for conducting cost-effectiveness analyses is growing, although it does not have a comparable mandate to NICE for recommending drugs for routine use.

However, international experience also shows that comparative clinical and cost-effectiveness assessments are not a panacea. In England, the combination of high prices and marginal clinical benefits associated with cancer drugs has resulted in fewer new drugs meeting NICE's cost-per-QALY threshold. Instead of denying coverage for a growing share of new drugs, policymakers have devised new mechanisms such as the Cancer Drugs Fund to cover drugs that do not meet NICE's cost-effectiveness criteria (Jack, 2014). The Cancer Drugs Fund is a so-called managed entry agreement - a contract between a manufacturer and a payer to allow market access under certain conditions, e.g., while additional data collection is underway. Such agreements are now common. Two thirds of OECD countries have introduced managed entry agreements to address the large uncertainty associated with early market access, most commonly for cancer drugs (Wenzl and Chapman, 2019). Despite their widespread use, the value of these arrangements to patients and health care systems is unclear. An evaluation of the English Cancer Drugs Fund found that most eligible drugs provided no measurable clinical benefit (Aggarwal et al., 2017).

Similar to the state laws identified by Leopold and colleagues, these recent payer-led policies can inadvertently compound existing uncertainties about cancer drug benefits. Increased use of special coverage arrangements for drugs with uncertain evidence has not incentivized higher-quality evidence 
generation. While managed entry agreements can require additional data collection to confirm clinical benefit, these arrangements have little prospect to produce robust evidence. Data collection efforts are rarely prespecified, seldom include clinical outcomes, and are limited by administrative constraints. In England, "real-world" studies were planned to address major uncertainties about the survival benefits of drugs included in the Cancer Drugs Fund, but these studies did not have prospective statistical analysis plans, potentially jeopardizing their validity (Macdonald and Goldacre, 2020). Evaluations of similar arrangements in Belgium and Sweden concluded that payer-mandated data collection efforts rarely resolved uncertainty around the effectiveness of new medicines (Wenzl and Chapman, 2019).

Leopold et al.'s study advances our understanding about how targeted cancer drug coverage laws can complicate decision making for payers. As calls for payment reform gain traction in the US, policymakers should consider the important unintended consequences of coverage policies. Experience to date suggests that targeted coverage may have further lowered already-limited incentives for manufacturers to produce high-quality evidence on their products. Recent attempts to manage uncertainty through tailored market entry agreements may have resulted in yet more uncertainty about the effectiveness of these drugs. These consequences matter not only to payers, but also to patients and their physicians, who need high-quality evidence to inform treatment decisions. 


\section{References}

Aggarwal, Ajay, T. Fojo, C. Chamberlain, C. Davis, and R. Sullivan. 2017. "Do Patient Access Schemes for High-Cost Cancer Drugs Deliver Value to Society?-Lessons from the NHS Cancer Drugs Fund." Annals of Oncology 28 (8): 1738-50. https://doi.org/10.1093/annonc/mdx110.

Aitken, Murray, Michael Kleinrock, Deanna Nass, and Alana Simorellis. 2019. "Global Oncology Trends 2019: Therapeutics, Clinical Development and Health System Implications.”

Blume-Kohout, Margaret E, and Neeraj Sood. 2013. "Market Size and Innovation: Effects of Medicare Part D on Pharmaceutical Research and Development." Journal of Public Economics 97 (January):32736. https://doi.org/10.1016/j.jpubeco.2012.10.003.

Chambers, James D., David D. Kim, Elle F. Pope, Jennifer S. Graff, Colby L. Wilkinson, and Peter J. Neumann. 2018. "Specialty Drug Coverage Varies Across Commercial Health Plans In The US." Health Affairs 37 (7): 1041-47. https://doi.org/10.1377/hlthaff.2017.1553.

Dakin, Helen, Nancy Devlin, Yan Feng, Nigel Rice, Phill O’Neill, and David Parkin. 2015. “The Influence of Cost-Effectiveness and Other Factors on Nice Decisions." Health Economics 24 (10): 1256-71. https://doi.org/10.1002/hec.3086.

Dusetzina, Stacie B., Haiden A. Huskamp, and Nancy L. Keating. 2019. "Specialty Drug Pricing and Outof-Pocket Spending on Orally Administered Anticancer Drugs in Medicare Part D, 2010 to 2019." JAMA - Journal of the American Medical Association 321 (20): 2025-27. https://doi.org/10.1001/jama.2019.4492.

Hernandez, Inmaculada, Alvaro San-Juan-Rodriguez, Chester B. Good, and Walid F. Gellad. 2020. "Changes in List Prices, Net Prices, and Discounts for Branded Drugs in the US, 2007-2018." JAMA 323 (9): 854-62. https://doi.org/10.1001/jama.2020.1012.

Jack, Andrew. 2014. “Which Way Now for the Cancer Drugs Fund?” BMJ 349: g5524.

Leopold, Christine, Rebecca L Haffajee, Christine Y Lu, and Anita K. Wagner. 202o. "The Complex Cancer Care Coverage Environment - What Is the Role of Legislation? A Case Study from Massachusetts." The Journal of Law, Medicine E Ethics.

Light, Donald W, and Joel Lexchin. 2015. "Why Do Cancer Drugs Get Such an Easy Ride?: Rushed Approvals Result in a Poor Deal for Both Patients and Cancer Research.” BMJ (Online) 350. https://doi.org/10.1136/bmj.h2o68.

Macdonald, Helen Poppy, and Ben Goldacre. 2020. "Does The Reformed Cancer Drug Fund Generate Evidence On Effectiveness? A Cross-Sectional Analysis On Publicly Accessible Documentation.” MedRxiv, March, 2020.03.06.19014944. https://doi.org/10.1101/2020.03.06.19014944.

MacLeod, Tatjana E., Anthony H. Harris, and Ajay Mahal. 2016. "Stated and Revealed Preferences for Funding New High-Cost Cancer Drugs: A Critical Review of the Evidence from Patients, the Public and Payers." The Patient - Patient-Centered Outcomes Research 9 (3): 201-22. https://doi.org/10.1007/s40271-015-0139-7.

Sommers, Benjamin D., and Aaron S. Kesselheim. 2018. "Massachusetts' Proposed Medicaid Reforms Cheaper Drugs and Better Coverage?” New England Journal of Medicine 378 (2): 109-11. https://doi.org/10.1056/NEJMp1714824. 
Stern, Ariel D, Felicitas Pietrulla, Annika Herr, Aaron S Kesselheim, and Ameet Sarpatwari. 2019. "The Impact of Price Regulation on the Availability of New Drugs in Germany." Health Affairs 38 (7): 1182-87. https://doi.org/10.1377/hlthaff.2018.05142.

Wenzl, Martin, and Suzannah Chapman. 2019. "Performance-Based Managed Entry Agreements for New Medicines in OECD Countries and EU Member States: How They Work and Possible Improvements Going Forward." OECD Health Working Papers, no. 115. https://doi.org/10.1787/6e5e4cof-en.

Wieseler, Beate, Natalie McGauran, and Thomas Kaiser. 2019. "New Drugs: Where Did We Go Wrong and What Can We Do Better?” BMJ 366: 14340. https://doi.org/10.1136/bmj.l4340.

Zhang, Audrey D., Jeremy Puthumana, Nicholas S. Downing, Nilay D. Shah, Harlan M. Krumholz, and Joseph S. Ross. 2020. "Assessment of Clinical Trials Supporting US Food and Drug Administration Approval of Novel Therapeutic Agents, 1995-2017." JAMA Network Open 3 (4): e203284. https://doi.org/10.1001/jamanetworkopen.2020.3284. 